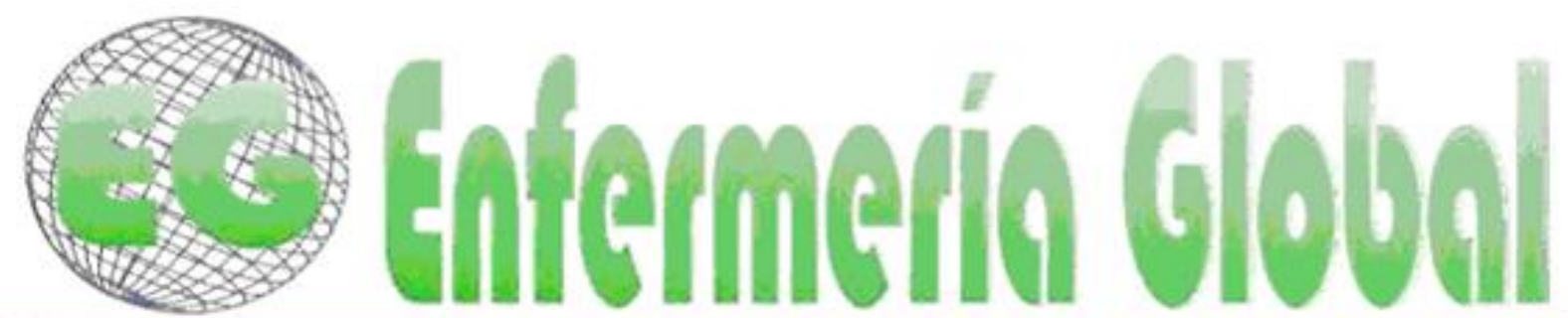

www.um.es/egloball

\title{
REVISIONES
}

\section{La gestión de los cuidados enfermeros en el hospital San Juan de Dios de Alicante: a propósito de tres fuentes documentales inéditas del siglo XVIII}

Management of nursing care in the San Juan de Dios hospital in Alicante: about three unpublished documentary sources of century XVIII

\section{*Núñez del Castillo, María de las Mercedes **Vizcaya Moreno, M Flores **Pérez Cañaveras, Rosa M}

\author{
*Adjunta de Enfermería del Hospital Universitario de San Juan de Alicante. Profesora Asociada del \\ Departamento de Enfermería, Universidad de Alicante. E-mail: mmnc@ua.es ** Profesora Titular de \\ Universidad. Departamento de Enfermería, Universidad de Alicante. Grupo de Investigación de
} Enfermería Clínica, Universidad de Alicante, Alicante, España.

Palabras clave: Gestión hospitalaria; Enfermería; San Juan de Dios; fuentes documentales; historia;
siglo XVIII Keywords: Hospital management; Nursing; San Juan de Dios; documentary sources; history; XVIII century

\section{RESUMEN}

Se propone como objetivo principal mostrar y analizar tres fuentes documentales inéditas del siglo XVIII, localizadas en el Archivo Municipal Sant Joan d'Alacant que aportan información sobre la gestión de enfermería realizada en el Hospital San Juan de Dios de Alicante. Son tres las fuentes documentales que se transcriben y analizan, una "relación jurada de Fray Joseph Martínez Maza", fechando el documento entre 1710 y 1718; un "Real privilegio de S.M. para amortizar la cantidad de 120 libras libres de sello y demás". Dado en 23 de enero de 1794, a favor del convento hospital de N.P. San Juan de Dios de la ciudad de Alicante y un "formulario o modelo para dar cumplimiento a la orden del nuncio de su Santidad referente a las rentas, gravámenes, limosnas y otras entradas, con la curación a pobres, enfermos, con arreglo a los libros de caja", datando el documento entre 1748 y 1760.

\section{ABSTRACT}

It is proposed as the main objective to display and analyse three unpublished documentary sources of the eighteenth century, located in the Municipal Archives of Sant Joan d'Alacant (Spain). These historic documents provide information on nursing management, held at the San Juan de Dios Hospital in Alicante. Three documentary sources are transcribed and analysed: 1) a sworn statement of Fray 
Joseph Martinez Maza, dating between 1710 and 1718; 2) a royal privilege of His Majesty in order to repay the amount of 120 pounds and others. Given at January 23, 1794, in favour of the convent hospital NP San Juan de Dios city of Alicante, and 3) a form to comply with the order of the Papal Nuncio of His Holiness relating to income taxes, alms and other inputs, with healing the poor, sick, under books cash document dating between 1748 and 1760 .

\section{INTRODUCCIÓN}

El hecho de cuidar, gestionar y administrar el hospital-convento de San Juan Bautista, como era conocido por su advocación más popular, por los Hermanos de San Juan de Dios, en la ciudad de Alicante, se remonta al año $1652^{1,2}$. Desde la fundación del hospital, los religiosos continúan con el ideal de su fundador teniendo entre sus funciones la gestión y asistencia de enfermería a los enfermos y pobres ${ }^{3}$. Esta gestión cotidiana del convento-hospital ha supuesto una tarea compleja desde los inicios de la Institución, y la búsqueda y generación de recursos económicos ha preocupado constantemente a los responsables de los centros hospitalarios ${ }^{4}$.

En España se desarrolla la cultura asistencial religiosa por dos motivos importantes, el Concilio de Éfeso (300 d. C) y por las teorías de San Isidoro. El Concilio de Éfeso recomendaba que todas las diócesis tuvieran junto a las catedrales un hospital para acoger a pobres y enfermos ${ }^{5}$.De hecho, en 1333, se estableció en la villa de Alicante un hospital benéfico, localizado junto a la Catedral de San Nicolás, fundado por Bernardo Gomis, comerciante catalán, casado en Alicante, y que otorga en su testamento de 25 de abril de ese año su hacienda para fundar un hospital para pobres y peregrinos, se instaló en unas casas que poseía en la Villa-Nueva, la cual ocupaba una vertiente del Benacantil, entre la calle de San Nicolás en el oeste, la calle del Hospital en el este San Agustín ${ }^{6}$, limitando al norte con la calle Virgen de Belén y al sur con la calle Mayor; la actual calle Montegón nace de los solares del hospital; tenía unas dimensiones de unos novecientos metros cuadrados, denominándose este hospital de San Juan Bautista y permaneció en la misma ubicación hasta su derribo en 1849.

La administración de los bienes que legó el Sr. Gomis para fundar el hospital era llevada a cabo por los jurados del Concejo, el Dean y el Cabildo de la colegiata y los curas de Santa María. En el año 1652, decidieron que fueran los Hermanos de San Juan de Dios quienes se encargaran de la gestión de dicho hospital, que entregan a los religiosos el día 8 de mayo de $1653^{6,7}$ y, como afirma García ${ }^{8}$, se establece convento en dicho hospital, cambiando el nombre por el de San Juan de Dios ${ }^{9}$, aunque en la advocación de la Casa y en los documentos consultados aparece el de San Juan Bautista ${ }^{1}$, que era como se conocía a este hospital popularmente.

Juan Santos en su obra de 1715, narra “...Fundación del hospital y convento San Juan Bautista de la ciudad de Alicante.... En esta ciudad entró nuestra religión por los años 1652 a tomar posesión de un hospital... como su fundación era tan antigua... estaba muy menoscabado y deteriorado..." ${ }^{10}$. Los religiosos de la orden permanecieron en este edificio hasta la exclaustración en 1849, derribándose posteriormente dicho inmueble.

Los documentos que abordamos nos aportan información valiosa sobre el hospital San Juan Bautista de Alicante, a la vez que nos ayudan a reflexionar sobre la problemática que suponía su gestión y la actividad asistencial realizada. Dos de los documentos consultados parecen ser la consecuencia de problemas medioambientales como pueden ser las avenidas o grandes lluvias, o la consecuencia 
de la guerra de Sucesión sufrida en años anteriores; y el tercer documento era utilizado para la justificación de los medios que disponía el hospital-convento para su mantenimiento.

En el presente trabajo nos hemos propuesto describir y analizar las fuentes documentales identificadas, describiendo el significado de la función gestora que desarrolla enfermería en el siglo XVIII, buscando contribuir así a un mejor conocimiento de la historia de este hospital.

\section{MATERIAL Y MÉTODO}

Se exponen y analizan tres documentos de distinta índole, localizados en el Archivo Municipal de: Sant Joan d'Alacant. Una relación jurada de Fray Joseph Martínez Maza, prior del Convento- Hospital de San Juan de Dios de Alicante, sobre las ruinas y pérdidas en bienes muebles de dicho hospital, sin fecha; un Real privilegio de S. M. para amortizar la cantidad de 120 libras libres del sello y demás. Dado en 23 de enero de 1794, a favor del Convento hospital de N.P. San Juan de Dios de la Ciudad de Alicante; un formulario o modelo para dar cumplimiento a la orden del Nuncio de Su Santidad referente a rentas, gravámenes, limosnas y otras entradas con la curación a pobres, enfermos, con arreglo a los libros de caja, sin fecha.

En esta ocasión no interesan las cuestiones formales de los documentos investigados, sino su contenido, la información obtenida nos ayuda a pensar y reflexionar sobre las dificultadas que entrañaba la gestión de estos centros sanitarios.

Una vez localizados los documentos se ha procedido a la transcripción y análisis, extrayéndose la información sobre la gestión del convento-hospital.

La identificación de las unidades documentales, es la siguiente:

1. Relación jurada de Fray Joseph Martínez Maza, prior del Convento-Hospital de San Juan de Dios de Alicante, sobre las ruinas y pérdidas en bienes muebles de dicho hospital. (Figura1) 
(1) Figura 1: Relación jurada de Fray Joseph Martínez Maza, prior del Convento yHospitalordende NroPr, San Juan de Dios de Alicante,

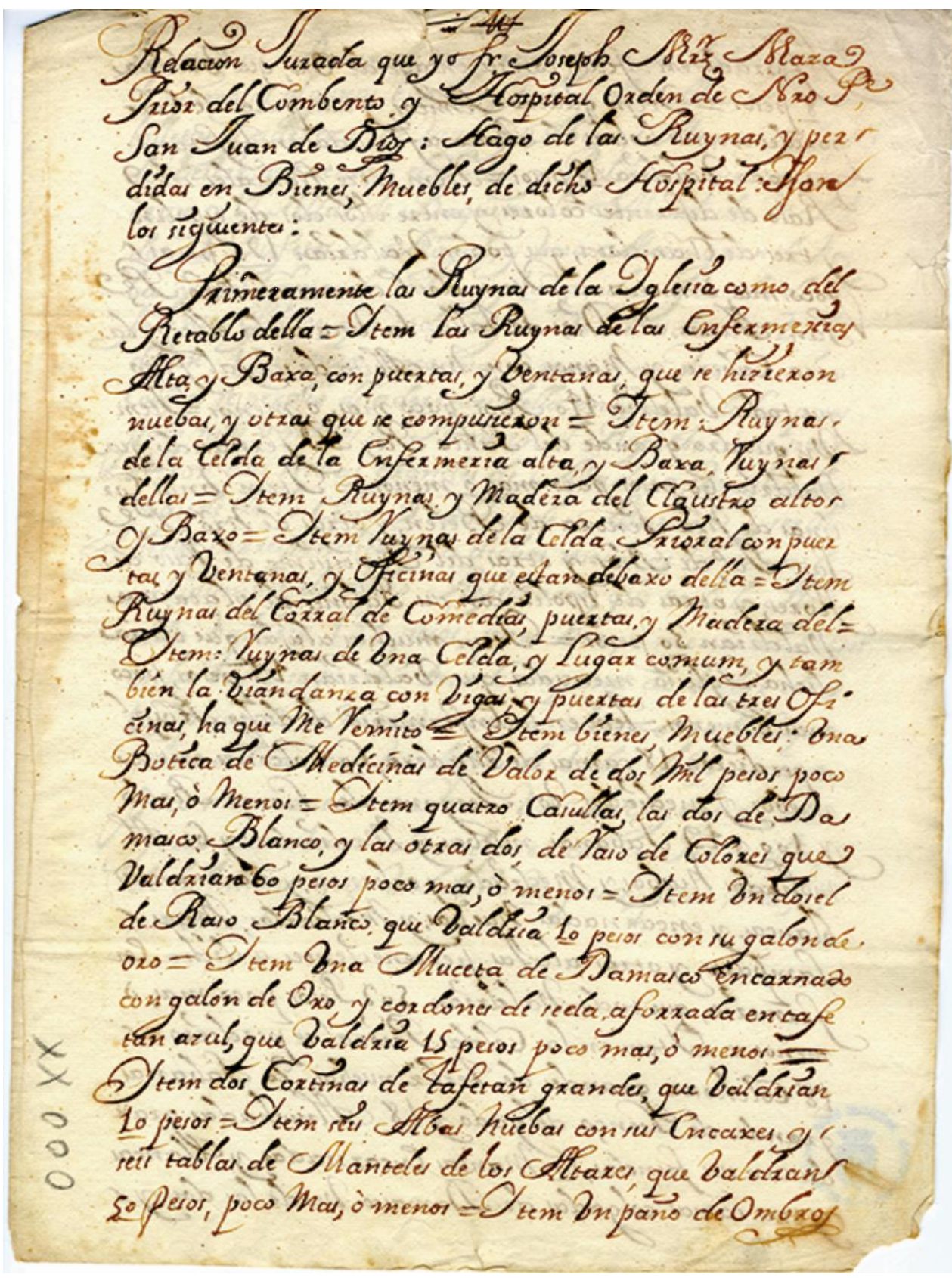
a. Signatura: $50 / 1-2$
b. $\quad$ Fondo/Colección: Colección Martínez - Mena.
c. Serie: Orden de San Juan de Dios
d. Fecha: sin fecha
e. Archivo: Municipal de Sant Joan d'Alacant
f. Volumen y soporte: papel 1 pliego, (3 pag. escritas), 213X304 mm. Manuscrito, castellano 
2. Real privilegio de S. M. para amortizar la cantidad de 120 libras libres del sello y demás. Dado en 23 enero de 1794, a favor del Convento hospital de N.P. San Juan de Dios de la Ciudad de Alicante (Figura 2)

(2) Figura 2: Real privilegio de S. M. para amortizar la cantidad de 120 libras libres del sello y demás.

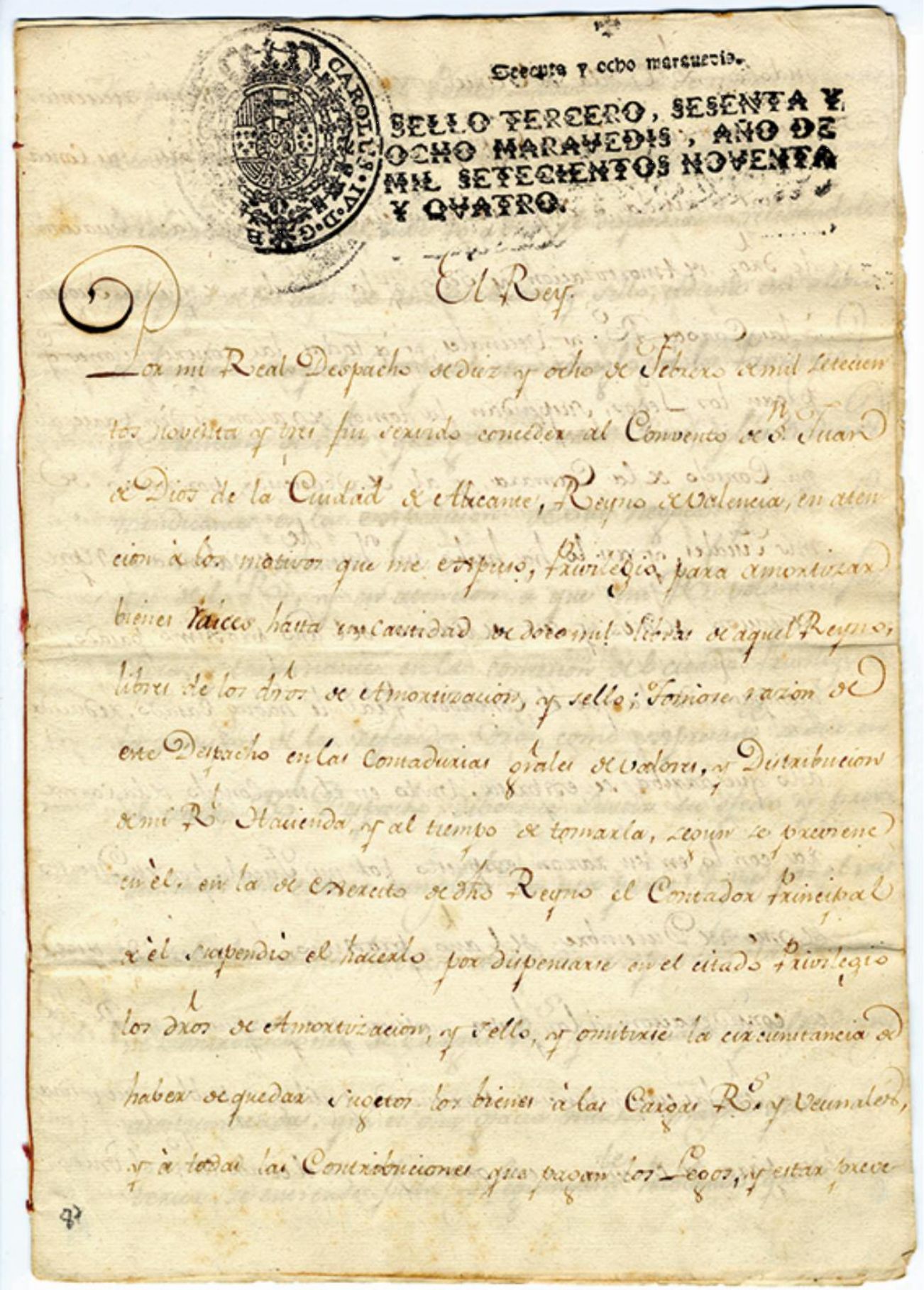

a. Signatura: 50/1 - 6

b. Fondo/Colección: Colección Martínez - Mena.

c. Serie: Orden de San Juan de Dios

d. Fecha: 1794, enero, 23, Aranjuez; 1794, marzo, 31, Alicante.

e. Archivo: Municipal de Sant Joan d'Alacant

f. Volumen y soporte: papel, 3 pliego, (12 págs.), 214×350 mm., cosidos; Manuscrito, castellano. 
3. Formulario o modelo para dar cumplimiento a la orden del Nuncio de Su Santidad referente a las rentas, gravámenes, limosnas y otras entradas, con la curación a pobres, enfermos, con arreglo a los libros de caja. (Figura 3)

(3) Figura 3: Formulario o modelo para dar cumplimiento a la orden del Nuncio de Su Santidad referente a las rentas, gravámenes, limosnas y otras entradas

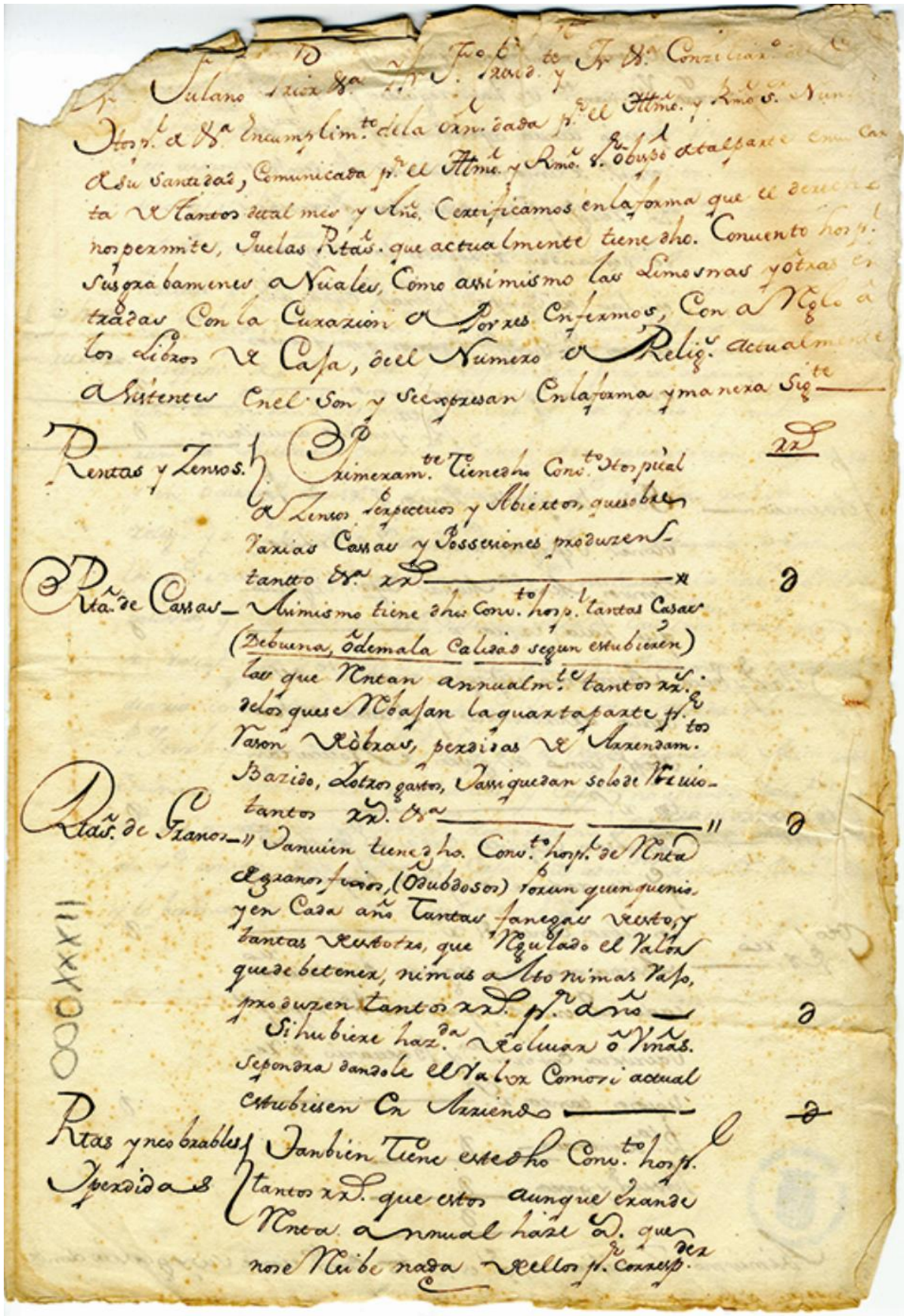
a. Signatura: $50 / 1-2$
b. Fondo/Colección: Colección Martínez - Mena.
c. Serie: Orden de San Juan de Dios
d. Fecha: sin fecha
e. Archivo: Municipal de Sant Joan d'Alacant
f. Volumen y soporte: papel 1 pliego, (3 págs. escritas), 214×310 mm. Manuscrito, castellano. 


\section{RESULTADOS}

"Relación jurada de Fray Joseph Martínez Maza, prior del Convento y Hospital orden de $\mathrm{NroP}^{r}$,San Juan de Dios de Alicante, sobre las ruinas y pérdidas en bienes muebles de dicho hospital."

(1) Relación jurada de Fray Joseph Martínez Maza, prior del Convento y Hospital orden de $\mathrm{NroP}^{\mathrm{r}}$, San Juan de Dios de Alicante,

Lo primero son las dudas que plantea el documento en la datación, para poder determinar el tipo de problemas que tuvo el convento-hospital en este tiempo y que condiciona la relación jurada.

"Relación Jurada que yo fr Joseph Mrz. ${ }^{n}$ Maza Prior del Convento. YHospital Orden deNroP', San Juan de Dios: Hagodelas Ruinas, y perdidas en Bienes, Muebles, de dicho Hospital: Y sonlos siguientes: Primeramente las Ruynas de la Iglesia como del Retablo della= Item de las ruynas de las Enfermerias..."

Fray Joseph Martínez Maza aparece por primera vez en los Capítulos Intermedios celebrados por la Congregación Española (1608-1833), en el Capítulo Intermedio, provincia de Castilla, celebrado en Madrid, el día 4 de mayo de 1710 aparece como prior del convento-hospital San Juan Bautista de Alicante José Maza; en el Capítulo Intermedio, provincia de Castilla, celebrado en Madrid, el día 4 de mayo de 1713 aparece como prior del convento-hospital San Juan Bautista de Alicante José Martínez Maza, siendo sustituido en el Capítulo General de 6 de mayo de 1718 por el prior Sebastián Montero ${ }^{11}$.

Ya sabiendo la fecha de dicha relación jurada, lo siguiente es presuponer la causa que justifica dicha relación, Ramos ${ }^{12}$ afirma que el bombardeo de 1691 en Alicante, originó grandes destrozos, y los combates de 1706 y 1709 entre ingleses y franceses por la posesión de la ciudad abocaron en la destrucción de buena parte de los edificios urbanos entre ellos el convento-hospital San Juan de Dios; en 1711, los trabajos de desescombro no habían concluido, en 1716, todavía perduraban los efectos de la destrucción; y autores más recientes como De la Torre ${ }^{10}$, verifica que con fecha de 8 de enero de 1716, hay una Resolución Capitular para que al hospital se le entregaran diez libras por vía de limosna, en atención a haber presentado en un memorial su notoria pobreza.

Haciendo referencia a este problema, Fray Juan Santos ${ }^{13}$, en su obra "Cronología Hospitalaria" impresa en 1716, habla de la llegada de los religiosos a Alicante, nos dice: "... Hoy pueden poco, y el hospital padece mucho por la invasiones que ha padecido la ciudad en los finales del siglo pasado, y en los principios de éste, de que le ha tocado gran parte al pobre hospital...".

En cuanto a la gestión de enfermería realizada por los religiosos de San Juan de Dios, entre 1710 y 1718 , teniendo en cuenta el inventario descubierto, el hospital tenía una capacidad entre 14 y18 camas entre mujeres y hombres, pudiendo aumentar su capacidad en caso de pestes, enfermedades, hasta un máximo de 40 o incluso 50 pacientes. Situación que desbordaría todos recursos disponibles para una buena gestión hospitalaria. Este número de camas quedaría confirmado por un documento de Cabildo de 24 de Marzo de 1733, donde manifiesta el Prior que "...solo hay puestas 18 camas para otros tantos enfermos que son los que tiene obligación de 
tener..."Y el resto de las camas, además de poder utilizarlas en caso de emergencia como se ha indicado anteriormente, el hospital además tiene "...sala aparte para hospedaje de pasajeros y peregrinos..."13.

“...Item de las ruynas de las Enfermerias Alta, y Baixa, con puertas, y Ventanas, que se hizieron nuevas, $y$ otras que se compusieron= Item: Ruynasde la celda de la Enfermeria Alta, y Baixa,..."

“...Item en la Enfermeria de dicho Hosp.', se perdieron 18 camas de madera, 60colch onesentre Nuebos y medianos, 20 Jergones de Lienço RecioY 100y 49 sabanas, nuevas y mediadas, y $\underline{60 A}$ Imohadasnuevas y mediadas, y ochenta, $y$ dos mantasblancas y encarnadas nuevas y mediadas, Mesas, Lamparasy otras atadas pertenecientes a dicha Enfermeria, ..."

“...Un Botica de Medicinas deValor de dos mil pesos poco mas, ò menos=..."

Se desconoce el número de religiosos que tenía la comunidad del convento, pero en el momento de la entrega del hospital, fueron un prior y un sacerdote y "... luego fueron más religiosos y se formó bastante comunidad para la asistencia de los enfermos..."13, en el análisis de la declaración jurada, en la celda prioral se pierden 18 colchones grandes, ropa de cama, y 12 sillas de baqueta, ¿era éste el número de religiosos que formaba la comunidad? De la Torre ${ }^{1}$ indica que el número de camas en 1833 era de 24, 200 pacientes asistidos y 6 religiosos, por lo que se puede aventurar, que ese sería el número de religiosos que habitualmente tenía el convento-hospital y que las otras camas podrían estar destinadas a los pasajeros o peregrinos que tenían acogida en el centro, o para otros religiosos que enviaba la ordena reforzar la comunidad en los momentos de emergencias; esta circunstancia se confirma en dos apartados del último documento analizado(formulario), donde dice:“......pero en abiendo .............de enfermos sepiden ael sup. ${ }^{\text {or }}$ g. ${ }^{r}$ los envía: ...." "y "...y en ocasion. ${ }^{s}$ de pestes, y Epidemias, $q .^{e}$ se pidenrelig. ${ }^{s}$ alaRelig. ${ }^{n}$.

Item en la Celda Prioral se perdieron 18 colchones grandes de ¿Terliz? Nuevos 20 sabanasgrandes de lienço delgado y 18 almohadas grandes con sus fundas, 10 Mantas encarnadas y blancas y tres camas colgadas de Damasco verde las dos, y La otra de Damasco encarnada con sus colchas de lo mismo, y algunas thoallas, tablas de mantel servilletas, y aderezos de ropa, y ¿H?Abitos, Mantas yRopa de vestir que tehianel Prior yReligiosos que entonceseran, y 12 sillas de baqueta de ¿Moscovia? Mediadas, 2 Bufetes de Nogal, y una mesa grande nueba, y tres camas de tablas, y 10 quadros grandes de diferentes efigies,que todo según Una conciencia Valdrían 1302 pesos , poco mas, ò menos. =

También se aprecia que se ha perdido el corral de comedias“...ltem Ruynas del Corral de Comedias,puertas, y Maderadel=..."que había junto al hospital, en unos patios adyacentes, y había sido inaugurado en 1616 con fines lucrativos-benéficos para el mantenimiento del Convento-Hospital, por tanto en1719,se ha de reconstruir la Casa de las Comedias del hospital por los daños producidos como consecuencia de la guerra de Sucesión ${ }^{15}$;el 23 de junio, 11 de noviembre de $1719 y 11$ de septiembre de 1723, en "Resolución Capitular de esas fechas, conceden permiso al Prior del Hospital para una representación de comedias $^{10}$.

La administración de la casa de las comedias era responsabilidad de los hermanos de San Juan de Dios, aunque para poder representar tenían que pedir permiso al 
Ayuntamiento. Este Corral de comedias producía beneficios importantes, para el sustento del convento-hospital y eran causa de disputas entre consistorio y hospital.

(2) "Real privilegio de S. M. para amortizar la cantidad de 120 libras libres del sello y demás. Dado en 23 enero de 1794, a favor del Convento hospital de N.P. San Juan de Dios de la Ciudad de Alicante".

El hecho de que el rey hiciera frente con una cantidad de dinero ante situaciones de emergencia no era infrecuente, ya que este documento no es el único del que se tiene constancia, García ${ }^{16}$, muestra que Felipe IV en 1649 , se vio precisado a librar treinta mil ducados para socorrer la ciudad de Málaga por el mal estado en que había quedado la hacienda de la ciudad por la peste que había sufrido. Y según el documento del Archivo Municipal de Alicante, hacia 1767, el Hospital San Juan de Dios quedó desbordado, por tener más de 30 ingresados; y el prior aseguró solo tener obligación de mantener 10 camas, por lo que también se pidió ayuda para la Institución ${ }^{17}$.

"... Visto en el mi Consejo de la Camara con lo en su razón expuesto por mi Fiscal, por su Decreto de once de Diciembre del año próximo, ha estimado que en consideración à los justos motivos que propuso à $m i R^{L}$ perdona ¿.......? las circunstancias denecesidad y utilidad publica que mediaban para la concesión desta gracia por ser el citado conv.$^{\text {to }}$ el único Hospital que hay en aquel puesto de mas donde se reciben enfermos de todas clases ocupando en ocasiones los claustros, y ante caso para la colocación de camas, tuve yo a bien el dispensárselas relevándole del pago delos $D c^{h}$ os de Amortizacion, $y$ Sello, cediendo este alivio en beneficio daquel Hospital con arreglo à los Privilegios y exencion deDchosque gozan estas Casas, y la Ordenes mendicantes..."

A pesar de las limitaciones que se plantean, las posibles situaciones de emergencia por las que el Rey concede este privilegio, pudieron ser debido a varias, una de ellas sería debido a situaciones de desbordamiento asistencial relacionadas con epidemias o fiebres, sobre todo terciarias. Alberola ${ }^{18}$, afirma que en 1783 , se inicia la epidemia en Alicante, su génesis pudo estar ocasionada por las fiebres tifoideas habituales en los ejércitos, a las que se unieron durante los años 1785 y 1786 una epidemia de calenturas terciana; también esta presión asistencial con saturación de camas podría ser debida a que era el único hospital que funcionaba en estos momentos tal como indica el documento, ya que el otro centro, denominado Hospital del Rey o militar, se utilizó para atender a soldados enfermos, y solo funcionaba cuando las necesidades lo requerían, pues las condiciones de salubridad no eran las mejores. La otra causa posible se relacionaría con abundantes lluvias e inundaciones que se produjeron a finales del siglo XVIII; en la Crónica de Alicante de Juan Bautista Maltés, hace referencia a una gota fría el 7 de noviembre de 1792, "... (1793)...el Rey se ha enterado... del riesgo que padecieron el convento de las religiosas Capuchinas de Alicante, como también los almacenes y edificios del barrio de San Francisco, de resultas de una copiosa lluvia...", convento localizado cerca del hospital, las inundaciones producidas por aguaceros de alta intensidad, dejaron sentir sus efectos destructivos de manera reiterada durante el último cuarto del siglo XVIII ${ }^{19}$. 
(3) "Formulario o modelo para dar cumplimiento a la orden del Nuncio de Su Santidad referente a las rentas, gravámenes, limosnas y otras entradas, con la curación a pobres, enfermos, con arreglo a los libros de caja".

Entre las condiciones puestas por los Patronos Jurados de la ciudad de Alicante a los religiosos para gestionar el hospital, fue la de rendir cuentas a estos patronos de dos en dos años de la hacienda que recibieron (especie de inventario) y de la que pudieran recibir en adelante ${ }^{6}$.

En este documento, se aprecia que el prior actuaba como verdadero gestor del hospital, realizando dicho inventario de los bienes.

"Fulano Prior(signo indescifrable).......J. Fr Presid. ${ }^{t e}$ y Fr. Consiliari del Hospital de (mismo signo indescifrable). Encumplim. ${ }^{\text {to }}$ de la orden dada por el p.r Iltmô y Rvdo. S. Nunciode su Santidad, comunicada p. 'el Iltmô. Y Rmô. Sr. Obispo de tal parte en sucartade tantos de tal mes y Año, Certificamos enlaforma que el derechonos permite, quelas Rentas que actualmente tiene el Convento Hospit.'sus gravámenes anuales, como asimismo las limosnas y ôtras entradas con la curación aPobres Enfermos, con arreglo âlos Libros de caja, del numero de Religiosos actualmenteasistentes en el. Son y se expresan e la form escritoa y manera sig ${ }^{\text {te }}$--------(posible valor?)"

No aparece la fecha de su elaboración, pero en el apartado del documento, "limosnas y entradas", no aparecen los importantes ingresos que se obtienen con la explotación de Corral de comedias, debido seguramente a que cuando estaba en uso este formulario o modelo, fue en el periodo en que se prohibieron sus representaciones por un Real

Decreto del 27 de julio de 1748, renovándose la prohibición en 1750, y no es hasta el 9 de abril de 1760, que por una orden Real se vuelve a autorizar las representaciones 15. Por lo que se podría datar entre el 27 julio de 1748 y el 9 de abril de 1760.

Limosnas y entradas. Ultimam. ${ }^{t e}$ se han regulado $p . . . . . . . . . . . . .$.

Que mis.......... acosta diferencia, $q^{e}$ se reser. ${ }^{v a}$ este

hosp.'de limosnas diarias, $q .{ }^{e}$ recogen

los relig. ${ }^{s}$. ${ }^{\prime}$ las calles, las q. ${ }^{e}$ reciben

de demandar, de legados, las de granos,

en las de campos, y otras, cada año,

tantos $s^{i}$ ?aunq. ${ }^{\text {e }}$ algunos quinquenios

suben mas, $y$ en otros menos.

$R^{\text {ta }}$ y entradas anuales. $-\ni------$

Benavides ${ }^{20}$, afirma que el patrimonio cultural de la Orden se ha ido generando a lo largo de la historia, y tiene un doble origen, por un lado las donaciones a la institución y por otro por el ejercicio ordinario de su actividad o lo que denomina mecenazgo. En el siglo XVIII, los hospitales se mantienen mediante una economía y un control riguroso, como se confirma en la transcripción de la fuente documental consultada, teniendo rentas y censos de casas, posesiones, rentas de grano, limosnas, también generaban pérdidas y tenían pagos.

Así mismo queda expresado en dicho documento, que los gestores del hospital se encargaban del gasto de botica, pagaban sus tributos, salarios, se mencionan 
expresamente el de los escribientes y oficiales del hospital, médico, cocinero y lavandera, Ilama la atención que aparece como asalariado del convento hospital un médico. Perdiguero ${ }^{11}$ señala que el cargo de médico asalariado de la ciudad de Alicante, tenía entre sus funciones la asistencia a los pobres enfermos del Hospital de San Juan de Dios, ¿pagaba el hospital otro médico para mejorar la asistencia?, posiblemente, ya que en el siglo XVIII, muchos de los médicos asalariados descuidaban la asistencia a los pobres enfermos, produciéndose continuas quejas por este motivo.

En los libros de caja que hace mención el documento hay un apartado muy importante a la gestión propiamente dicha de las enfermerías, dando constancia del número de camas, de los pacientes vistos en el año y número de religiosos.

“...tantas salas de Enfermerías contantas camas, en las quales segúnconsta en el asiento de los Libros ¿secuscan? tantos pobres enfermos ...... cada año ( si hay ¿ingresos?,) cada al.assis.... las enfermerías, pr. ${ }^{q}$ sino .... enferm. ${ }^{e}$ y los hombresp. ${ }^{r}$ tantos relig. ${ }^{s} q .{ }^{e}$ en el num. Señalado $p .^{r}$ la religión $p .^{a}$ lo otro: $y$ entre ellos un sacerdote para la adm. ${ }^{\text {on }}$ de los s. ${ }^{\text {tos }}$ sacram. ${ }^{\text {tos }}$ y en el $q .{ }^{e}$ regularm. ${ }^{\text {te }}$ tiene esta casa aezepcion de uno, ô dos, $q{ }^{e}$........pero en abiendo ...de enfermos sepiden ael sup. ${ }^{\text {or }}$ g.'los envía:

Llama la atención que el problema de falta de personal y la necesidad de personal de refuerzo ante situaciones de emergencia o saturación, no es un tema nuevo, ya que en este documento queda constancia que ante estas situaciones se pedían religiosos para reforzar la asistencia a los pacientes.

“...y también se curan los militares enferm. ${ }^{s} q .{ }^{e}$ suele aber guarnicion, ô depasso y en ocasion. ${ }^{s}$ de pestes, y Epidemias, $q .{ }^{e}$ se pidenrelig. ${ }^{s}$ ala Relig. ${ }^{n}$ (orden) y a p. ${ }^{\text {es? }}$ S.M. (Dios leg. ${ }^{e}$ )..."

\section{CONCLUSIONES}

Trascritos y analizados estos tres documentos, se ha llegado a la conclusión: que la gestión de enfermería era una parte muy importante en la vida cotidiana del hospital de San Juan de Dios de Alicante durante el siglo XVIII.

Por desgracia, los documentos encontrados y analizados son fragmentarios y dispersos, aunque rescatan del pasado información muy importante para el conocimiento del funcionamiento del hospital Convento de san Juan de Dios, así como su gestión.

Ha resultado interesante, poder llegar a la conclusión de la fecha en la que se realizó la relación jurada, que es entre el 4 de mayo de 1710 y el 3 de mayo de 1718, considerando que José Maza y José Martínez Maza son las mismas personas, ya que en la relación jurada aparece como Joseph Mrz. ${ }^{n}$ Maza; y en el caso de que no lo fueran, podemos datar el documento entre el 5 de mayo de 1713 y 5 de mayo de 1718.

Así mismo podemos decir que la causa que justifica la relación jurada fue como consecuencia de la guerra de Sucesión; y el origen que justifica el Real privilegio de S.M a favor de convento hospital de N.P. San Juan de Dios de la ciudad de Alicante, 
se pudo deber a situaciones de desbordamiento asistencial relacionadas con epidemias o fiebres, sobre todo terciarias, aunque también esta presión asistencial con saturación de camas podría ser debida a que era el único hospital que funcionaba en estos momentos tal como indica el documento; siendo la otra causa posible la relacionada con abundantes lluvias e inundaciones que se produjeron a finales del siglo XVIII.

En cuanto al formulario para dar cumplimiento de rentas y gravámenes, se podría datar entre el 27 julio de 1748 y el 9 de abril de 1760 y muestra el control exhaustivo que se llevaba en los libros de caja.

\section{FUENTES}

Documento: Relación jurada de Fray Joseph Martínez Maza, prior del ConventoHospital de San Juan de Dios de Alicante, sobre las ruinas y pérdidas en bienes muebles de dicho hospital. Sin fecha. Signatura: 50/1 Archivo: Municipal de Sant Joan d'Alacant

Documento: Real privilegio de S. M. para amortizar la cantidad de 120 libras libres del sello y demás. Dado en 23 enero de 1794, a favor del Convento hospital de N.P. San Juan de Dios de la Ciudad de Alicante. Signatura: 50/1 - 6. Fecha: 1794, enero, 23, Aranjuez; 1794, marzo, 31, Alicante. Archivo: Municipal de Sant Joan d'Alacant

Documento: Formulario o modelo para dar cumplimiento a la orden del Nuncio de Su Santidad referente a las rentas, gravámenes, limosnas y otras entradas, con la curación a pobres, enfermos, con arreglo a los libros de caja. Sin fecha. Signatura: 50/1 - 2.Archivo: Municipal de Sant Joan d'Alacant

\section{Agradecimiento}

A Don Jorge Payá, archivero del Municipio de Sant Joan d'Alacant en el momento de realizarla búsqueda, por la ilusión puesta en el proyecto y las facilidades dadas.

\section{REFERENCIAS}

1. Torre de la Rodríguez, F. Estadística de los conventos y religiosos de las provincias juandedianas en España en 1833. Exclaustración y Orden Hospitalaria: estado de la cuestión (I) .Archivo hospitalario 2004; (2): 21-194.

2. García Hourcade, J J. Los Hermanos de San Juan de Dios en el sureste español: Estrategias fundacionales y problemas (siglo XVI-XVII). Archivo Hospitalario 2006; (4) 475-489.

3. Miralles Sangro MT, Duran Escribano M. El milagro de San Juan de Dios, de Murillo. Cultura de los cuidados. 2006;X (20) 50-54.

4. Benavides Vázquez F. Gestión hospitalaria y relaciones con la administración. A propósito de un documento de conciliación entre el hospital General de Madrid y el hospital de Antón Martí (1799). Archivo hospitalario2011; (9) 361-373.

5. Fernandes de Freitas G, Siles González J. Antropología y cuidados en el enfoque de San Juan de Dios. Index Enferm [revista en la Internet]. 2008 Jun [citado 2012 Sep 16]; 17(2): 144-148. Disponible en: http://dx.doi.org/10.4321/S11321296200800015

6. Martínez San Pedro R. Historia de los hospitales en Alicante. Instituto de Estudios alicantinos. Diputación Provincial de Alicante. (Patronato José Ma ${ }^{a}$ Quadrado del C.S.I.C.).Serie 1, n.16. 1974. 
7. Perdiguero Gil E. "Con medios humanos y divinos": La lucha contra la muerte y la enfermedad en Alicante en el siglo XVIII. Dynamis.2002. Acta. Hisp. Sci. Hist.lllus. (22). 121-150.

8. García HourcadeJ J. Los Hermanos de San Juan de Dios en el sureste español: Estrategias fundacionales y problemas (ss. XVI-XVII). Archivo hospitalario 2006; (4) 475-489.

9. Vivarens y Pastor R."Crónica de la muy llustre y siempre fielciudad de Alicante" 1876 .Reproducción facsímil publicada en el centenario de la misma como homenaje a su autor. ISBN: 84-400-9913-14. 1976.

10. Torre de laRodríguez F. Panorámica de la provincia de Nuestro Padre San Juan de Dios, de Castilla, en vísperas de la exclaustración de 1835: Cartas-cuenta e inventarios de los conventos-hospitales remitidos al capítulo General intermedio a celebrar el 3 de mayo de 1833, en el Convento-HospitalDe Nuestra Señora del Amor de Dios y Venerable Padre Antón Martín, de Madrid. Exclaustración y Orden Hospitalaria: Estado de la cuestión (VI). Archivo hospitalario 2010; (8) 17-231.

11. Torre de la Rodríguez F. Elencos de los Capítulos Generales e Intermedios celebrados por la Congregación Española (1608-1833). Disponible en:www.sanjuandediso-fic.org/mm/file/portal\%20juandediano/2.2.2.pdf-(Consultado 2 septiembre 2012).

12. Ramos Hidalgo A. (1984): Desarrollo urbano de Alicante: La vila nova $\begin{array}{llll}\text { medieval. HISTORIA Medieval1984 (3) 277-287.Disponible } & \end{array}$ en:http://hdl.handle.net/10045/7144.Consultado 19-06-2012.

13. Santos J. Chronología HospitalariaDisponible en: ]2.3.1.juan santos oh - Orden Hospitalaria de San Juan de ...Consultado 24 noviembre 2014

14. LLoret Esquerdo J. La casa de les Comèdies d'Alacant (1616-1793) En: El Teatre catalá: dels origens al segle XVIII/Albert Rosich, Antoni Serra, Pep Valsalobre (eds) Kassel: Edition Reichenberger (estudis catalans; 5) 2001 . 333-345. ISBN 978-3935004-34-3.Dispoible en:La Casa de les Comèdies d'Alacant (1616-1793) RUAconsultado20-11-2014

15. García Monlleó J, Girón Irueste F. La asistencia de los Hermanos de San Juan de Dios en las epidemias andaluzas (siglos XVI a XIX). Archivo hospitalario2005 (3) 545-569.

16. Perdiguero Gil E, Bernabeu Mestre J. La asistencia médica pública en el Alicante del siglo XVIII: los médicos de la ciudad. Canelobre, revista del Instituto de Cultura "JuanGil-Albert" 1995(29-30) 165-176.

17. Alberola Romá A.Una enfermedad de carácter endémico en el Alicante del siglo XVIII: Las fiebres terciarias. Revista historia moderna 1985 (5) 127-140. Ed. Universidad Alicante. ISSN 0212-5862.

18. Alberola Romá A. Riadas, inundaciones y desastres en el sur valenciano a finales del siglo XVIII. Papeles de geografía, 2010 (51-52)23-32 ISSN: 0213-1781.

19. Benavides Vázquez F. El concepto de patrimonio cultural en la Orden Hospitalariade San Juan de Dios. Origen y evolución histórica. Archivo hospitalario 2010(8) 261-285.

Recibido: 15 de marzo 2015; Aceptado: 13 de mayo 2015

ISSN 1695-6141

๑ COPYRIGHT Servicio de Publicaciones - Universidad de Murcia 